\title{
Does ampullary adenoma size predict invasion on EUS? Does invasion on EUS predict presence of malignancy?
}

Authors

Institutions
Vaishali Patel ${ }^{1}$, Paul Jowell' ${ }^{1}$, Jorge Obando ${ }^{1}$, Cynthia D. Guy ${ }^{2}$, Rebecca A. Burbridge ${ }^{1}$

Division of Gastroenterology, Duke University Medical Center, Durham, North Carolina, United States

${ }^{2}$ Division of Pathology, Duke University Medical Center, Durham, North Carolina, United States submitted

14. October 2015

accepted after revision

24. October 2016

\section{Bibliography}

DOI http://dx.doi.org/

10.1055/s-0042-121001

Endoscopy International Open

2016; 04: E1313-E1318

(c) Georg Thieme Verlag KG

Stuttgart · New York

E-ISSN 2196-9736

Corresponding author Vaishali Patel, MD

558 Rock Springs Road NE Atlanta, GA 30324

Phone: +1-610-780-5874

vapatel83@gmail.com
Background and study aims: It is common practice to perform ampullectomy without endoscopic ultrasound (EUS) for ampullary lesions $<1 \mathrm{~cm}$ but no data exists to support it. No studies have explored whether EUS findings of invasion correlate with malignancy or high-grade dysplasia (HGD) on pathology. We explored the association between adenoma size, pathology results, and invasion on EUS.

Patients and methods: This was a single-center retrospective cohort study at a large tertiary care academic hospital. Chart review was performed for 161 patients with benign ampullary lesions on endoscopic biopsy (identified by pathology records). The primary outcomes were mean size $(\mathrm{mm})$ of adenomas and pathology findings with and without intraductal and/or duodenal wall invasion on EUS.

\section{Introduction}

$\nabla$

Ampullary tubular (TA) and tubulovillous adenomas (TVA) can undergo malignant transformation [1] and their recognition and appropriate management is therefore imperative. Endoscopic ampullectomy (EA) is a preferable option for lesions that are not invasive due to lower morbidity compared to surgical ampullectomy (SA) [2-5]. It is challenging to determine the presence of invasion based on endoscopic characteristics alone [6], but it is imperative to exclude invasion of the duodenum involving the submucosa (stage T1b), invasion beyond the muscularis propria (stage T2), or involvement of the pancreas/pancreatic duct (PD) or common bile duct (CBD), as these are risk factors for incomplete endoscopic resection, lymph node metastasis, recurrence or presence of adenocarcinoma [7-10]. Presence of invasion is considered an indication for SA [11 - 15].
Endoscopic ultrasound (EUS) has good accuracy for T-staging of ampullary adenocarcinoma, and
Results: Invasion was identified by EUS in 41 (34.1\%) of 120 patients who underwent EUS. The mean size of the lesion in these patients was $20.9 \mathrm{~mm}( \pm 11.6 \mathrm{~mm})$ compared to $13.9 \mathrm{~mm}$ $( \pm 11.3 \mathrm{~mm}, P=0.0001)$ in patients without invasion. A receiver operating characteristic (ROC) curve (AUC 0.73, 95\% CI 0.63-0.83) revealed 100 $\%$ sensitivity for absence of invasion on EUS in lesions less than $6.5 \mathrm{~mm}$. Invasion on EUS had sensitivity of $63.0 \%$ (95\% CI $47.0 \%-77.0 \%)$ and specificity $88.0 \%$ (95\% CI $78.0 \%-95.0 \%$ ) for presence of malignancy, HGD or invasion on pathology. Conclusions: EUS should be considered for ampullary lesions $>6.5 \mathrm{~mm}$. This study provides evidence to support the practice of ampullectomy without EUS for smaller adenomas. EUS evidence of invasion is highly specific for pathologic malignancy, HGD, or invasion (which preclude endoscopic ampullectomy).

good sensitivity/specificity for assessment of invasion compared to endoscopic retrograde cholangiopancreatography (ERCP) and pathology results [16]. However, no data exist to guide which ampullary lesions should undergo EUS prior to ampullectomy. ASGE guidelines recommend generally considering EUS before resection, while others suggest that lesions $<1 \mathrm{~cm}$ without suspicious features of malignancy may not require ultrasound prior to removal [17-19]. The $1-\mathrm{cm}$ size cutoff is based on expert opinion, and there are no data to guide whether the size of adenoma can predict the presence of invasion, or if it can help determine the need for EUS prior to EA. Therefore, practices regarding pre-ampullectomy EUS vary between centers. Furthermore, while invasion on EUS has been shown to correlate with extension on pathology, there are no studies exploring whether invasive characteristics on EUS predict the presence of malignancy or HGD on pathology. 
161 patients with ampullary adenomas (median size $13 \mathrm{~mm}$, IQR $9-20 \mathrm{~mm}$ )

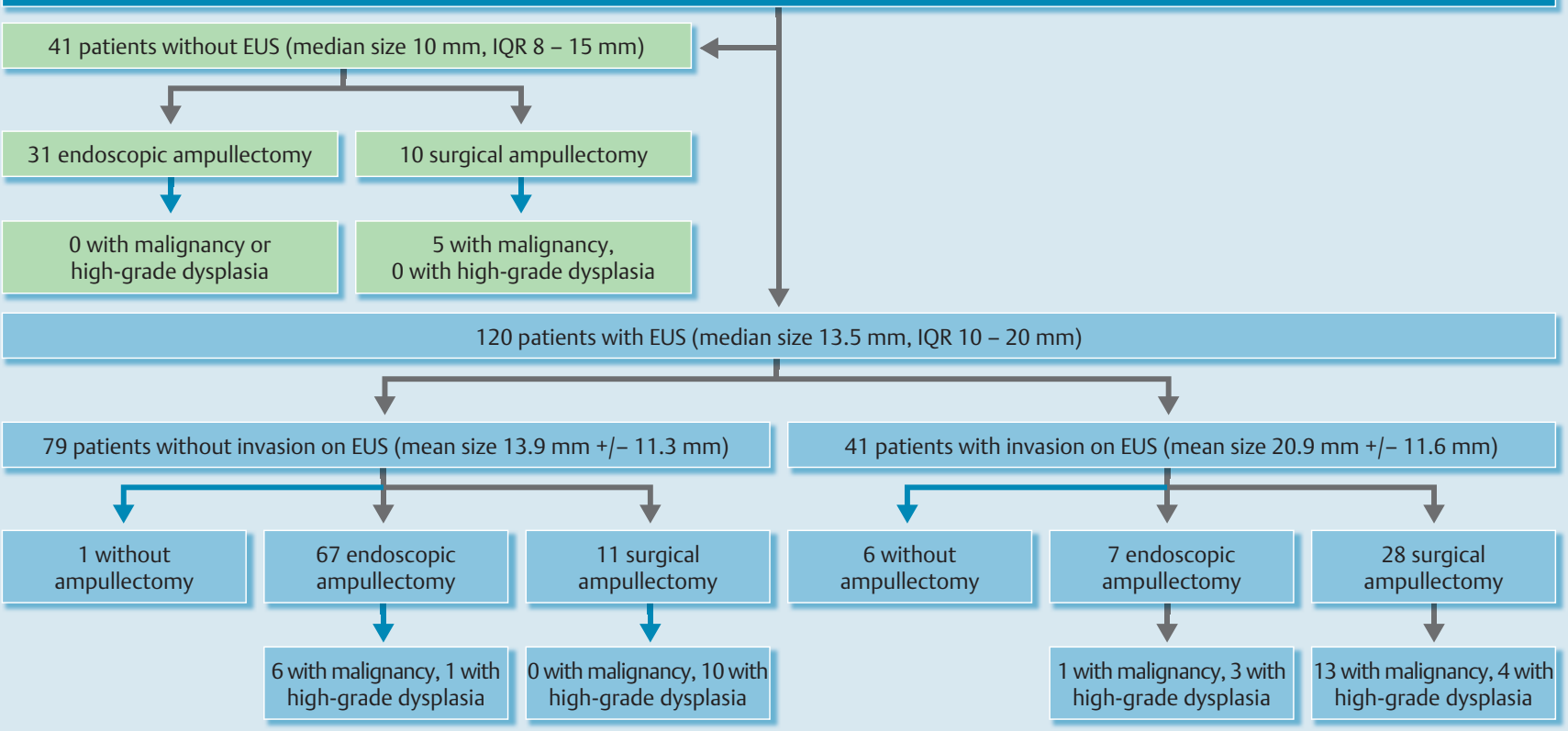

Fig. 1 Patient cohort with ampullary adenomas between January 2000 and December 2011.

Our purpose was to determine whether adenoma size differs between ampullary adenomas with invasive characteristics on EUS compared those without invasion. This is the first study to address this issue. It provides evidence for the size criteria to support the practice of some centers to proceed to EA without EUS for small adenomas. Secondarily, this study reports the sensitivity and specificity of invasion on EUS for malignancy, HGD, or invasion on pathology.

\section{Patients and methods}

$\nabla$

\section{Study population}

We identified all patients with benign ampullary lesions (TA and TVA, including low and HGD) on endoscopic biopsy between January 2000 and December 2011 at Duke University Medical Center by pathology records ( $n=161, \diamond$ Fig. 1 ). Patients with malignancy revealed on initial biopsy or pre-EUS stent placement were excluded. Chart review was performed for size of the mass (size of specimen after ampullectomy on pathology report), endoscopic appearance as noted by the endoscopist when available, EUS characteristics, method of ampullectomy, final pathology results, adverse events associated with ampullectomy within the first year, recurrence of adenomatous tissue, and management of recurrence. Presence of invasion was defined as any CBD or PD intraductal (ID) invasion (regardless of length) with or without CBD dilation, or involvement of the duodenal submucosa (SM) or deeper. Patients for whom ID or SM invasion could not be definitively diagnosed by EUS were excluded. Final pathology results following ampullectomy were reviewed for size of lesion, presence of adenocarcinoma, HGD, and evidence of ID/SM invasion.

\section{EUS examination}

Patients underwent either upper endoscopy or ERCP prior to EUS, which prompted the referral for EUS. The decision to refer for EUS was made by the treating endoscopist based on availability of
EUS at our center and on the appearance or size of the lesion, or based on the endoscopist's discretion for proceeding to ampullectomy without EUS ( Fig. 2 shows an ampullary adenoma seen on endoscopy). The indication for EUS in all the patients was to perform locoregional staging. All EUS examinations occurred prior to EA or SA. Pre-resection mucosal biopsies were done prior to EUS in all patients. All EUS procedures were performed at Duke University Medical Center by one of three same experienced endosonographers during the entire study period (advanced endoscopy attendings, R.B., P.J., or J.O.). Routine EUS was completed to assess tumor size, ID extension, ductal dilation and pancreas invasion, SM involvement or the muscularis propria, and regional lymph node involvement. Fig. 3 demonstrates an example of CBD invasion seen on EUS. EUS was performed using radial echoendoscopes (model EG-3670URK, Pentax Medical Co, Montvale, $\mathrm{NJ}$ ) and water instillation for improved visualization of the ampulla.

\section{Surgical or endoscopic ampullectomy}

The decision to proceed with EA or refer for SA was made by the treating endoscopist. The decision for ERCP followed by EA was at the discretion of the advanced endoscopist if the lesion was deemed amenable to resection. At our center, patients with invasive lesions (especially ID or pancreas invasion) were referred for $S A$ regardless of degree of invasion. A patient may also have been referred for EA of a lesion due to contraindications to SA. EA was performed via a side-viewing duodenoscope with a therapeutic working channel (model ED-3490TK Pentax Medical Co, Montvale, NJ). When feasible, en bloc resection was completed using electrocautery snare. Otherwise a piecemeal resection technique was used. Electrocautery or argon plasma coagulation ablation was done for suspected residual adenomatous tissue after resection. Pancreatic stents were placed when possible to reduce the risk of post-ERCP pancreatitis. SA was completed by either pancreaticoduodenectomy (Whipple's procedure) or transduodenal ampullectomy by surgeons at Duke University Medical Center or Duke Regional Medical Center. 

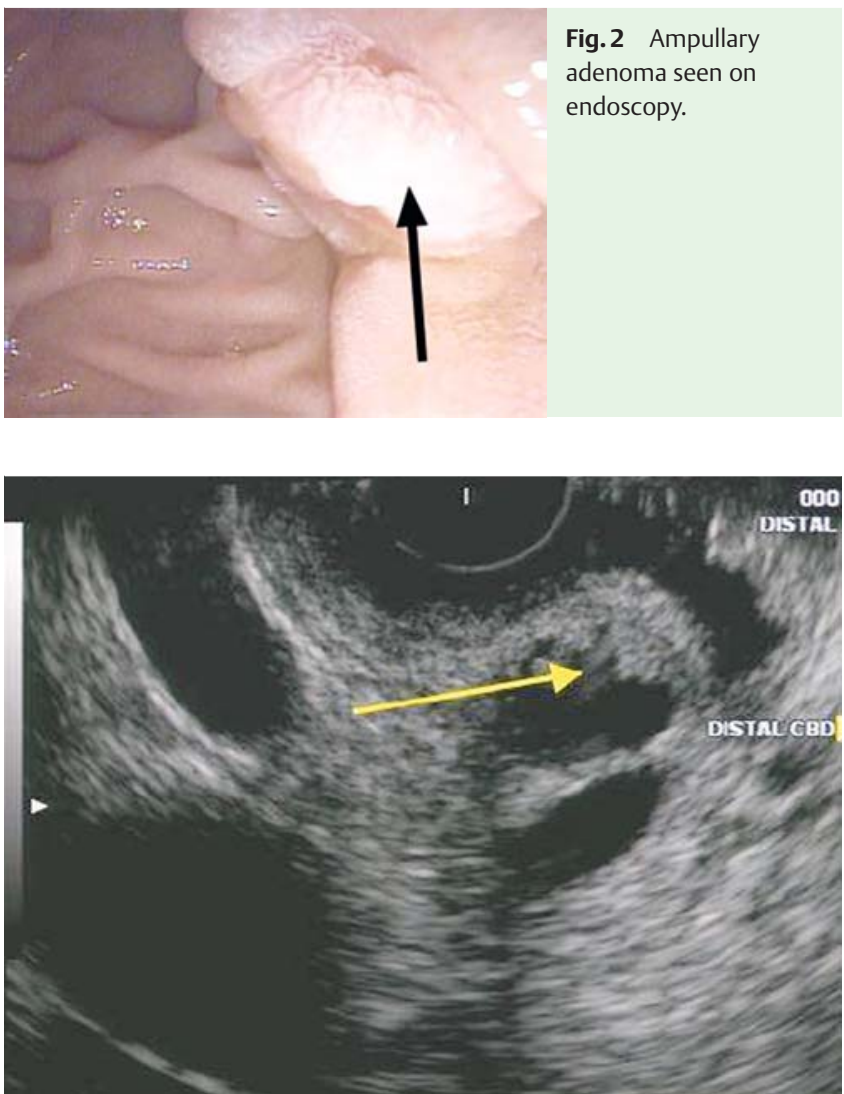

Fig.3 CBD invasion of an ampullary adenoma seen on EUS.

\section{Statistical analysis}

All statistical testing was performed using R Studio (2012, version 0.98.1028, Boston MA). Means and standard deviations were calculated for size of adenoma in each group. A power anal$\mathrm{ysis}$ was done using $\mathrm{R}$ for a difference in mean size of adenoma (in $\mathrm{mm}$ ) between lesions with invasive characteristics on EUS compared to those without. With an alpha value of 0.05 and $80 \%$ power in a two-sample $t$-test power calculation, it was found that 33 patients would be needed per arm to find a difference in means of at least $7 \mathrm{~mm}$. (We determined this was a reasonable estimation for a clinically significant size difference that could be noted at time of endoscopy and could prompt EUS evaluation of an adenoma. There is no existing literature upon which this estimation could be based). The Wilcoxon rank sum test with continuity correction was performed to compare the mean size of mass between those that had invasive characteristics on EUS and those that did not. An ROC curve was created to evaluate sensitivity and specificity of size as a predictor for absence of invasive characteristics on EUS with associated AUC, size thresholds, and confidence intervals were found. The sensitivity and specificity of invasion on EUS to predict malignancy, HGD, or invasion on pathology were calculated.

\section{IRB statement}

Approval was obtained from the Duke University Medical Center Institutional Review Board for this study (Pro00042641).

\section{Results}

$\nabla$

\section{Patient and adenoma characteristics}

The mean age of the 161 patients with benign ampullary adenomas was 59.1 years $( \pm 16.2$ years). Ninety patients $(55.9 \%)$ were female. The median size of the lesions was $13 \mathrm{~mm}$ (interquartile range $9 \mathrm{~mm}-20 \mathrm{~mm})$. Ninety-nine patients $(61.8 \%)$ had lesions greater than $10 \mathrm{~mm}$. Thirty-seven $(23.0 \%$ ) patients had a known polyposis syndrome. The indication for the endoscopy that diagnosed the adenoma was "surveillance for adenomas" in these patients. In the remainder of the patients, ampullary adenomas were found incidentally during upper endoscopy or ERCP done for other indications. The indications for endoscopy were abdominal pain (33 patients, $20.5 \%$ ), iron deficiency anemia ( 7 patients, $4.3 \%$ ), gastrointestinal bleeding (14 patients, $8.7 \%$ ), polyposis surveillance (37 patients, $23.0 \%$ ), dysphagia (5 patients, $3.1 \%$ ), and gastroesophageal reflux (10 patients, $6.2 \%$ ). Indications for ERCP were abnormal liver enzymes (30 patients, $18.6 \%$ ), choledocholithiasis or cholangitis (11 patients, $6.8 \%$ ), and pancreatitis (14 patients, $8.7 \%$ ).

\section{Patients without EUS evaluation}

Mean age of the 41 patients who did not undergo EUS evaluation was 50.3 years ( \pm 16.4 years). Median lesion size in this group was $10 \mathrm{~mm}$ (interquartile range $8 \mathrm{~mm}-15 \mathrm{~mm}$ ). They proceeded to either EA if felt amenable by the advanced endoscopist (31 patients [75.6\%], mean age 46.3 years \pm 16.1 years, with mean lesion size of $9.2 \mathrm{~mm}, \pm 3.7 \mathrm{~mm}$ ) or SA if the endoscopist felt the lesion was too large for EA (10 patients [24.4\%], mean age 62.2 years \pm 10.9 years, with mean lesion size of $32.3 \mathrm{~mm} \pm 21.3 \mathrm{~mm}$ ). Malignancy was found in 5 (12.2\%) SA patients. Among SA cases, adverse events (AEs) of bleeding, wound infection or dehiscence occurred in 6 patients (60.0\%). Two patients $(20.0 \%)$ had recurrence of malignancy (liver or lymph node metastasis detected 3 years and 8 years after surgical resection. Both patients had no metastatic disease on pre-operative cross-sectional imaging). Among the $31 \mathrm{EA}$ patients, 4 (12.9\%) had AEs consisting of postampullectomy bleeding, possible perforation, mild pancreatitis, or an ampullary stricture requiring stent placement; there was recurrence of adenomatous tissue on biopsy in 6 patients (19.4\%) treated with APC or resection.

\section{Patients with EUS evaluation}

One hundred twenty of 161 (74.5\%) patients underwent EUS evaluation. Their mean age was 62.0 years ( \pm 15.5 years). Median lesion size was $13.5 \mathrm{~mm}$ (interquartile range $10 \mathrm{~mm}-20 \mathrm{~mm}$ ). Of these 120 patients, $101(84.2 \%)$ underwent ERCP at our center. There was agreement between EUS and ERCP with regard to characteristics of invasion in $96(95.0 \%)$ patients. EUS was positive for invasion when ERCP was not in 4 (4.0\%) patients. ERCP suggested invasive characteristics in $1(1.0 \%)$ patient, which were not seen on EUS. Among the 19 patients who did not undergo ERCP, 10 patients (52.6\%) had been referred directly for EUS, and then referred to SA upon finding invasive characteristics on EUS. Two patients (10.5\%) opted for surveillance and 1 was lost to follow-up (no ampullectomy performed). Six patients (31.6\%) were directly referred for surgery without ERCP due to the endoscopist feeling the lesion was not amenable to EA despite not having EUS characteristics of invasion (see below).

Thirty-nine of the 120 (32.5\%) EUS patients underwent SA, and $74(61.7 \%)$ patients had EA. Seven patients (5.8\%) did not undergo ampullectomy, either due to patient preference, age or comor- 
bidities precluding surgical ampullectomy, or loss to follow-up. Malignancy or HGD was found in 38 patients (31.7\%) among these 120 patients. Of the 74 EAs, there were AEs of minor postpolypectomy hemorrhage in 9 patients (12.2\%), and a retroperitoneal perforation and biliary fistula in 1 patient (1.4\%). Of the 39 patients with SA, 9 patients $(23.1 \%$ ) had AEs of infection, pancreatitis, duodenal leak (one patient), and prolonged ileus (two patients).

\section{Patients without EUS characteristics of invasion}

There were 79 (65.8\%) patients without EUS characteristics of invasion. Eleven (13.9\%) patients underwent SA due to the endoscopist feeling the lesion was not amenable to EA (mostly due to size of lesion, irregular borders and inability to exclude duodenal invasion, or abutment of the CBD without definite invasion). Mean lesion size in these 11 patients was $28.9 \mathrm{~mm} \pm 23.3 \mathrm{~mm}$. Of the remaining 68 patients, 1 patient did not undergo EA (repeat biopsy showed normal tissue in one of these patients, and the patient was kept under surveillance), and the rest underwent EA (mean size $11.3 \mathrm{~mm} \pm 5.3 \mathrm{~mm}$ ). Nine of the $67(13.4 \%)$ EAs were complicated by gastrointestinal bleeding, with 5 of them requiring endoscopy with or without intervention (3 were minor hemorrhages). One patient had a biliary fistula with retroperitoneal perforation. Sixteen (23.9\%) patients experienced recurrence of adenomatous tissue after EA treated with argon plasma coagulation and surveillance; 1 of these patients was referred for surgery after 2 recurrences. Of the $11 \mathrm{SAs}$, there were AE of pancreatitis, prolonged ileus, or wound infection in $3(27.2 \%)$ patients. 2 (18.1\%) surgical patients experienced recurrence of adenomatous tissue, and 1 of them underwent repeat surgical resection. Adenocarcinoma was found in 6 of the $78(7.7 \%)$ patients who underwent ampullectomy (median size of lesion $15.5 \mathrm{~mm}$, IQR $13.5 \mathrm{~mm}-21.3 \mathrm{~mm}$ ); all of these patients had undergone EA. These patients were referred to surgery following return of the final pathology results showing malignancy. HGD was found in 11 patients (14.1\%). The remaining $61(78.2 \%)$ patients had TA or TVA without pathological evidence of invasion, HGD, or adenocarcinoma.

\section{Patients with EUS characteristics of invasion}

Forty-one of 120 (34.2\%) patients had invasion on EUS. Twelve patients (29.3\%) had both ID involvement in addition to SM involvement ( 3 of these patients had extension to the muscularis propria). 6 patients (14.6\%) had SM involvement only (5 patients involving the submucosa, 1 patient with involvement extending to the muscularis propria). 23 patients (56.1\%) had ID involvement only.

Seven (17.1\%) patients underwent EA due to patient age precluding surgery and patient preference. One of these 7 patients had adenocarcinoma on pathology after ampullectomy, and 3 had HGD (all of these had recurrence of adenomatous tissue on surveillance requiring argon-plasma coagulation treatment twice). Twenty-eight patients (75.6\%) underwent SA. Six patients (14.6\%) declined ampullectomy (SA was recommended for these patients). Six SA patients had AEs of pancreatitis, bowel perforation, bowel ischemia, and infection. Recurrence of adenomatous tissue occurred in 5 (18.5\%) surgical patients. Of the total 35 patients with invasion on EUS who underwent ampullectomy, 14 patients $(35.0 \%)$ had adenocarcinoma on final pathology, and 7 patients $(20.7 \%$ ) had HGD (total of 21 patients, $52.5 \%$ ). Six patients had TA or TVA with ID extension on pathology, but no HGD or adenocarcinoma. Five patients had adenomas with no
Table 1 Characteristics of patients with invasion vs. no invasion of ampullary adenoma on EUS.

\begin{tabular}{|c|c|c|}
\hline Patient characteristics & $\begin{array}{l}\text { No invasion on } \\
\text { EUS }(n=79)\end{array}$ & $\begin{array}{l}\text { Invasion on } \\
\text { EUS }(n=41)\end{array}$ \\
\hline Mean age, years (SD) & $59.5( \pm 16.2)$ & $66.7( \pm 11.7)$ \\
\hline Male gender & $41(51.8 \%)$ & $17(41.5 \%)$ \\
\hline Mean lesion size, mm (SD) & $13.9( \pm 11.3)$ & $20.9( \pm 11.6)$ \\
\hline Presence of polyposis syndrome & $18(22.8 \%)$ & $2(4.9 \%)$ \\
\hline Endoscopic ampullectomy & $67(84.8 \%)$ & $7(17.1 \%)$ \\
\hline Surgical ampullectomy & $11(13.9 \%)$ & $28(75.6 \%)$ \\
\hline $\begin{array}{l}\text { Malignancy or high-grade } \\
\text { dysplasia on final pathology }\end{array}$ & $17(21.5 \%)$ & $21(52.5 \%)$ \\
\hline
\end{tabular}

EUS: endoscopic ultrasound.

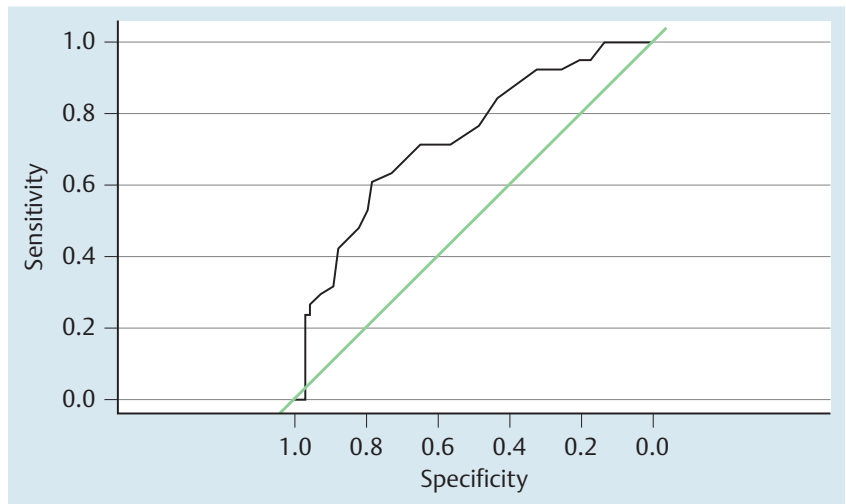

Fig. 4 Receiver operating characteristic (ROC) curve (AUC 0.73, 95\% Cl $0.63-0.83$ ) for size of ampullary adenoma and invasive characteristics on EUS.

pathological evidence of SM or ID invasion. Three patients had no adenomatous tissue on final pathology (normal tissue in 1 patient, acute inflammation in 2 patients).

Mean lesion size with invasion on EUS was $20.9 \mathrm{~mm}( \pm 11.6 \mathrm{~mm})$ compared to $13.9 \mathrm{~mm}( \pm 11.3 \mathrm{~mm}, P=0.0001)$ in those without invasion ( $\triangle$ Table 1 ). An ROC curve (AUC $0.73,95 \%$ CI $0.63-0.83$ ) revealed $100 \%$ sensitivity for absence of invasion on EUS in lesions less than $6.5 \mathrm{~mm}$ ( $\bullet$ Fig.4). The overall sensitivity for invasive findings on pathology, malignancy or HGD was $63.0 \%$ (95\% CI $47.0 \%-77.0 \%$ ) and specificity $88.0 \%$ (95\% CI 78.0\%-95.0\%). The positive predictive value was $77.0 \%(95 \% \mathrm{CI} 60.0 \%-90.0 \%)$ and the negative predictive value was $79.0 \%$ (95\%CI $68.0 \%-88.0 \%)$. The positive likelihood ratio was 5.42 (95\% CI $2.71-10.81)$. The negative likelihood ratio was 0.42 ( $95 \% \mathrm{Cl} 0.28-0.63)$. The sensitivity of EUS evidence of ID invasion (with or without SM involvement) for pathologic evidence of ID invasion, HGD or malignancy was $62 \%(95 \% \mathrm{CI} 46.0 \%-76.0 \%)$ and specificity is $95.0 \%(95 \% \mathrm{CI}$ $87.0 \%-99.0 \%$ ). The positive likelihood ratio was 13.21 (95\% CI $4.27-40.88)$.

\section{Discussion}

$\nabla$

Invasive characteristics on EUS may preclude EA. Previous studies have demonstrated the performance characteristics of EUS for local staging of ampullary adenocarcinoma. Existing data demonstrate its superiority compared to ultrasound, computed tomography, and magnetic resonance imaging and its accuracy of $90 \%$ when compared to surgical pathology results for assessing 
ID invasion [20-24]. While the practice at some centers is to proceed to ampullectomy without EUS for lesions $<1 \mathrm{~cm}$, this cutoff is based on anecdotal experience and expert opinion. It is unknown whether adeoma size can predict presence of invasive characteristics, and whether invasion on EUS correlates with malignancy on final pathology when preliminary biopsies are negative for adenocarcinoma. Studies have suggested that presence of invasion on EUS is a risk factor for occult malignancy, however, the significance of invasion on EUS with regard to histopathologic presence of malignancy or HGD remains unknown [25].

In our study, we assessed the association between the size of ampullary adenomas and presence of invasion on EUS. We found a statistically significant difference in lesion size between those with and without invasive characteristics on EUS. Furthermore, invasion on EUS was highly unlikely in lesions smaller than $6.5 \mathrm{~mm}$. Size criteria may therefore help to determine for EUS prior to endoscopic resection. Contrary to the current practice of ampullectomy without EUS for lesions $<1 \mathrm{~cm}$, our results suggest that EUS perhaps should be considered for adenomas between $5 \mathrm{~mm}$ and $10 \mathrm{~mm}$. Smaller lesions can likely be resected with a low risk of incomplete resection. This study also demonstrates that while invasion on EUS has moderate sensitivity (63.0\%) for malignancy on pathology, it has higher specificity (88.0\%). Furthermore, the specificity is even greater $(95.0 \%)$ for histopathologic presence of malignancy, HGD, or ID invasion for lesions with EUS evidence of ID involvement. This has clinical implications as presence of invasion on EUS rules in the likelihood of malignancy, HGD, or invasion on pathology (all of which would prompt surgical referral). Length of ID invasion was not consistently documented in our study and therefore was not a part of the analysis. However, at our institution, any degree of ID invasion is an indication for SA. While centers with high volume and experienced endosonographers may have satisfactory complete resection rates for lesions with $<1 \mathrm{~cm}$ ID extension, a conservative approach may otherwise be preferable to avoid incomplete resection or recurrence. These results may be useful in building an algorithm and developing society guidelines on the management of ampullary lesions. Interestingly, we found adenocarcinoma on final pathology in 6 of $83(7.2 \%)$ patients without EUS characteristics of invasion. One possible reason for this is under-staging of invasion by EUS (as has been demonstrated by previous series) $[26,27]$, or a very minute degree of invasion that was not clearly distinguishable on EUS. We found agreement between EUS and ERCP with regard to invasive characteristics in 96 of 101 (95.0\%) patients who had both procedures, which is comparable to previous studies [16]. Adenocarcinoma is often missed on initial forceps biopsies of the ampulla, and as such, malignancy was found on final pathology in 25 of the 153 (16.3\%) patients who underwent ampullectomy. This may have been due to sampling missing smaller foci of adenocarcinoma or ID areas inaccessible by forceps.

This study had several limitations. We presented data from a single center, and management of ampullary lesions based on size and degree of ID involvement may differ at other centers. Our dataset had a limited number of patients with only SM invasion (6 patients). A larger dataset containing more patients with SM involvement would allow for better assessment of the predictive characteristics of EUS in this population, especially because the degree of duodenal involvement impacts staging. In this study, there were no predefined criteria for performing EUS. As such, there was no uniform treatment protocol because of the retrospective nature of the study. Furthermore, patients with ampullary adenomas underwent ampullectomy without EUS due to the variability in referral, which decreased the overall number of patients undergoing EUS. By reviewing medical records over a longer time, we were able to have a sample size large enough to be adequately powered for our research question of interest. Future prospective studies with uniform protocols are needed in this field. The majority of patients with ID involvement (regardless of whether it was limited or extensive invasion) were referred for SA. One advantage of this was better consistency in referral patterns for SA versus endoscopic EA. Another limitation of the retrospective design of this study is missing data or inconsistencies in documentation of EUS findings between endoscopists regarding the endoscopic appearance of the ampullary mass that were suggestive of possible malignant transformation. Ulceration, friability, or puckering of the ampulla was present in 4 of 120 (3.3\%) patients, all of whom had EUS characteristics of invasion as well. It is unknown whether the appearance of the lesions in other patients with EUS characteristics of invasion was not concerning for malignant transformation, or whether the information was not documented in the report. This leads to an inherent limitation of the ROC analysis in that it does not address whether lesion size predicts ductal invasion independently, and tests for association and independence from size could not be completed. In addition, the exact size of the lesion by endoscopic or EUS appearance was not consistently available. As such, lesion size was obtained from specimen size as noted in the pathology report following ampullectomy for the sake of accuracy and consistency. Lesion size estimated by the endoscopist was not used given that it was not consistently documented in all of the EUS reports. Had that information been available consistently, using the endoscopist's estimation of the size would be closer to representing clinical practice. However, endoscopists' estimation of size is also largely subjective, and agreement between endoscopists is more likely to occur at the thresholds suggested by our study of $<5 \mathrm{~mm}$ versus $>5 \mathrm{~mm}$, or $>10 \mathrm{~mm}$, rather than between these values. Lastly, variability in the endoscopists' interpretation of invasion may have been introduced by improvement of processors and equipment over the course of the study. We feel that the degree of this variability in interpretation is likely small. of note, the complication rates found in our study are similar to those published in previous studies from our center [5].

While identification of invasion by EUS is important to reduce rates of difficult EA and incomplete resection, there are few data to direct which ampullary adenomas should undergo EUS evaluation. There are no data regarding whether adenoma size informs need for EUS, or whether invasion on EUS predicts malignancy on pathology. Our study identified the size threshold for performing EUS to diagnose invasion. In addition, invasion on EUS demonstrated high specificity for presence of malignancy, HGD, or invasion on pathology, all of which are reasons to consider referral for SA. These data have the potential to impact clinical practice and to help standardize management of benign ampullary lesions across centers, and potentially prevent unnecessary procedures for patients. Our study suggests the need for prospective studies to establish the significance of invasive characteristics on EUS, and to compare the outcomes of incomplete resection, recurrence, and presence of malignancy.

\section{Competing interests: None}




\section{References}

1 Hirota WK, Zuckerman MJ, Adler DG et al. ASGE guideline: the role of endoscopy in the surveillance of premalignant conditions of the upper GI tract. Gastrointest Endosc 2006; 63: 570-580

2 Jordan PH Jr, Ayala G, Rosenberg WR et al. Treatment of ampullary villous adenomas that may harbor carcinoma. J Gastrointest Surg 2002; 6: $770-775$

3 Cahen DL, Fockens $P$, de Wit $L T$ et al. Local resection or pancreaticoduodenectomy for villous adenoma of the ampulla of Vater diagnosed before operation. Br J Surg 1997; 84: 948 -951

4 Tran TC, Vitale GC. Ampullary tumors: endoscopic versus operative management. Surg Innov 2004; 11: 255 - 263

5 Ceppa EP, Burbridge RA, Rialon KL et al. Endoscopic versus surgical ampullectomy: an algorithm to treat disease of the ampulla of Vater. Ann Surg 2013; 257: 315-322

6 Castillo $C$. Endoscopic ultrasound in the papilla and the periampullary region. World J Gastrointest Endosc 2010; 2: 278-287

7 Ridtidid W, Tan D, Schmidt SE et al. Endoscopic papillectomy: risk factors for incomplete resection and recurrence during long-term followup. Gastrointest Endosc 2014; 79: 289-296

8 You D, Heo J, Choi $S$ et al. Pathologic T1 subclassification of ampullary carcinoma with perisphincteric or duodenal submucosal invasion: Is it T1b? Arch Pathol Lab Med 2014; 138: $1072-1076$

9 Bohnacker S, Seitz U, Nguyen D et al. Endoscopic resection of benign tumors of the duodenal papilla without and with intraductal growth. Gastrointest Endosc 2005; 62: 551 - 560

$10 \mathrm{Kim} \mathrm{HA,} \mathrm{Kim} \mathrm{KM,} \mathrm{Shin} \mathrm{JU} \mathrm{et} \mathrm{al.} \mathrm{Prediction} \mathrm{of} \mathrm{carcinoma} \mathrm{after} \mathrm{resection}$ in subjects with ampullary adenomas on endoscopic biopsy. J Clin Gastroenterol 2013; 47: 346-351

11 Bohnacker S, Seitz U, Nguyen D et al. Endoscopic resection of benign tumors of the duodenal papilla without and with intraductal growth. Gastrointest Endosc 2005; 62: 551 - 560

12 Seewald S, Omar S, Soehendra N. Endoscopic resection of tumors of the ampulla of Vater: how far up and how deep down can we go? Gastrointest Endosc 2006; 63: 789-791

13 Desilets DJ, Dy RM, Ku PM et al. Endoscopic management of tumors of the major duodenal papilla: refined techniques to improve outcome and avoid complications. Gastrointest Endosc 2001; 54: 202-208

14 Norton ID, Gostout CJ, Baron TH et al. Safety and outcome of endoscopic snare excision of the major duodenal papilla. Gastrointest Endosc 2002; 56: $239-243$
15 Catalano MF, Linder JD, Chak A et al. Endoscopic management of adenoma of the major duodenal papilla. Gastrointest Endosc 2004; 59: 225 232

16 Ridtitid W, Schmidt SE, Al-Haddad MA et al. Performance characteristics of EUS for locoregional evaluation of ampullary lesions. Gastrointest Endosc 2015; 81: 380 - 388

17 Zadorova Z, Dvofak M, Hajer J. Endoscopic therapy of benign tumors of the papilla of Vater. Endoscopy 2001; 33: $345-347$

18 Adler DG, Qureshi W, Davila R et al. ASGE guideline: The role of endoscopy in ampullary and duodenal adenomas. Gastrointest Endosc 2006; 64: $849-854$

19 Baillie J. Endoscopic ampullectomy. Am J Gastroenterol 2005; 100: $2379-2381$

20 Chen CH, Tseng LJ, Yang CC et al. The accuracy of endoscopic ultrasound, endoscopic retrograde cholangiopancreatography, computed tomography, and transabdominal ultrasound in the detection and staging of primary ampullary tumors. Hepatogastroenterology 2001; 48: 1750 1753

21 Artifon EL, Couto D Jr, Sakai P et al. Prospective evaluation of EUS versus CT scan for staging of ampullary cancer. Gastrointest Endosc 2009; 70: 290-296

22 Manta R, Conigliaro R, Castellani D et al. Linear endoscopic ultrasonography vs magnetic resonance imaging in ampullary tumors. World J Gastroenterol 2010; 16: 5592 - 5597

23 Chen $\mathrm{CH}$, Yang CC, Yeh YH et al. Reappraisal of endosonography of ampullary tumors: correlation with transabdominal sonography, $\mathrm{CT}$, and MRI. J Clin Ultrasound 2009; 37: 18 - 25

24 Trikudanathan G, Njei B, Attam R et al. Staging accuracy of ampullary tumors by endoscopic ultrasound: meta-analysis and systematic review. Dig Endosc 2014; 26: 617-626

25 Gaspar J, Shami $V$. The role of EUS in ampullary lesions: Is the answer black and white? Gastrointest Endosc 2015; 81: 389-390

26 Ito $K$, Fujita N, Noda $Y$ et al. Preoperative evaluation of ampullary neoplasm with EUS and transpapillary intraductal US: a prospective and histopathologically controlled study. Gastrointest Endosc 2007; 66: $740-747$

27 Azih LC, Broussard BL, Phadnis MA et al. Endoscopic ultrasound evaluation in the surgical treatment of duodenal and peri-ampullary adenomas. World J Gastroenterol 2013; 19: 511-515 